\title{
Acknowledgement for Reviewers for 2020
}

\section{Atilla Ansal ${ }^{1}$}

Published online: 2 March 2021

(C) The Author(s), under exclusive licence to Springer Nature B.V. part of Springer Nature 2021

The quality of any scientific journal depends to a large extent upon the voluntary work done by reviewers. This important work is usually done in anonymity. Considering the time and effort that is put into reviewing, the Editor-in-Chief, Associate Editors and Editorial Board of Bulletin of Earthquake Engineering acknowledge this by publishing a list of reviewers. The list below gives the names of all known reviewers of accepted and rejected manuscripts in 2020. We thank you all.

$\begin{array}{lll}\text { Adel Abdelnaby } & \text { Sara Amoroso } & \text { Anze Babic } \\ \text { Hassan Abedi Sarvestani } & \text { Hamidreza Anajafi } & \text { Mohammad Reza } \\ \text { Lars Abrahamczyk } & \text { Anthimos S. Anastasiadis } & \text { Bagerzadeh Karimi } \\ \text { Christoph Adam } & \text { Guido Andreotti } & \text { Laurie Baise } \\ \text { Naida Ademovic } & \text { Pavan Aninthaneni } & \text { Konstantinos Bakalis } \\ \text { Rohit Adhikari } & \text { Hideo Aochi } & \text { Jack Baker } \\ \text { Shrabony Adhikary } & \text { Georgios Apostolakis } & \text { Ihsan Engin Bal } \\ \text { Mahdi Adibi } & \text { Amjad Aref } & \text { Arnab Banerjee } \\ \text { Naveed Ahmad } & \text { Juana Arias-Trujillo } & \text { Daniele Baraldi } \\ \text { Maria Aiello } & \text { Danny Arroyo } & \text { Andre R. Barbosa } \\ \text { Habib Akbarzadeh Bengar } & \text { Carlos Arteta } & \text { Pierre-Yves Bard } \\ \text { Mitsuyoshi Akiyama } & \text { Payam Asadi } & \text { Carla Barnaba } \\ \text { Sinan Akkar } & \text { Arash Askari } & \text { Julien Baroth } \\ \text { Vahid Akrami } & \text { Panagiotis Asteris } & \text { Alberto Basaglia } \\ \text { Yasemin Didem Aktas } & \text { Anastasia Athanasiou } & \text { Dhiman Basu } \\ \text { M. Shahria Alam } & \text { Gail Atkinson } & \text { Josep Batllo } \\ \text { Guillermo Aldama Bustos } & \text { Samuel Auclair } & \text { Mahmoud Bayat } \\ \text { Alper Aldemir } & \text { Çigdem Avc1-Karataş } & \text { Alemdar Bayraktar } \\ \text { Reza Allahvirdizadeh } & \text { Stefano Aversa } & \text { Farhad Behnamfar } \\ \text { Joao Almeida } & \text { Özgür Avşar } & \text { André Belejo } \\ \text { Gülay Altay } & \text { A. Ayala } & \text { Andrea Belleri } \\ \text { Ahmet Can Altunişık } & \text { Ersin Aydin } & \text { Beatrice Belletti } \\ \text { Daniel Ambrosini } & \text { Alireza Azarbakht } & \text { Amadeo Benavent- } \\ \text { Gabriele Ameri } & \text { Raffaele Azzaro } & \text { Climent }\end{array}$

Atilla Ansal

atilla.ansal@ozyegin.edu.tr

1 Istanbul, Turkey 
Nassima Benmansour

Rita Bento

Alessandro Vittorio

Bergami

Mehmet Berilgen

Antonio Bernardo Sanchez

Carl Bernier

Luisa Berto

Etienne Bertrand

Bjarni Bessason

Michele Betti

Kaiming Bi

Nicoletta Bianchini

Christian Bignami

Samuel Bignardi

Ahm Muntasir Billah

Emilio Bilotta

Philippe Bisch

Dionysis Biskinis

Rajib Biswas

Carlos Blandon

Eden Bojórquez

Davide Bolognini

Julian Bommer

Paolo Boncio

Edmund Booth

Barbara Borzi

Stathis Bousias

Claude Boutin

Marco Bovo

Franco Braga

Giuseppe Brando

Miguel Bravo-Haro

Jan Bröchner

Brian Broderick

Emanuele Brunesi

Svetlana Brzev

Mladen Bulic

Zachary Bullock

Hilmar Bungum

Nicola Buratti

Oreste Bursi

Christoph Butenweg

Pierfrancesco Cacciola

Marcello Cademartori

Chiara Calderini

Ivo Calio'

Vítor Camacho

Alfredo Camara
Alp Caner

Donatello Cardone

Athol Carr

Claudia Caruso

Claudia Casapulla

Alessio Cascardi

Siro Casolo

Paolo Castaldo

Massimina Castiglia

Carlo Castiglioni

Giulio Castori

José Miguel Castro

Nicola Caterino

Serena Cattari

Liborio Cavaleri

Francesco Cavalieri

Mehmet Celebi

Oguz Cem Celik

Kemal Cetin

Bharat Chalise

Andrew Chanerley

Shu-Hsien Chao

Finley Charney

Robert Chase

Charisis Chatzigogos

Tushar Chaudhari

Hu Cheng

Claudio Chesi

Anna Chiaradonna

Kosuke Chimoto

Eugenio Chioccarelli

Eunsoo Choi

Christis Chrysostomou

Marios Chryssanthopoulos

Christophe Clement

Francesco Clementi

Paulo Coelho

Fabrizio Comodini

Michael Constantinou

Vincenzo Convertito

Dario Coronelli

Marco Corradi

António A. Correia

Maria Rosaria Costanzo

Anabella Cotovanu

Rita Couto

Gemma Cremen

Maria Crespo

Helen Crowley
Misko Cubrinovski

Alberto Cuevas

Shengai Cui

Jamal Dabbeek

Kaoshan Dai

Panos Dakoulas

Bruno Dal Lago

Giancarlo Dal Moro

Larentiu Danciu

Mohamed Darwish

Atefe Darzi

Shideh Dashti

Manuela Davi

Christopher de la Torre

Jose Vieira de Lemos

Flavia De Luca

Gianfranco De Matteis

Maria Teresa De Risi

Filomena de Silva

Mario De Stefano

Hervè Degee

Matthew DeJong

Ciro Del Vecchio

Marta Del Zoppo

Jose Delgado

Pedro Delgado

Derya Deniz

Antonio Di Cesare

Antonella Di Meo

Luigi Di Sarno

Fabio Di Trapani

Elias Dimitrakopoulos

Haiping Ding

Florea Dinu

Ricardo Dobry

Marco Donà

Baiping Dong

You Dong

John Douglas

Christophe Droz

Chunbo Du

Mojtaba Dyanati

Hossein Ebrahimian

Nurhan Ecemis

Eleftheria Efthymiou

Anaxagoras Elenas

Yasser Eljajeh

Ahmed Elkady

Alaa El-Din Elsisi 
Murat Altug Erberik

Bulent Erkmen

E. Ece Eseller-Bayat

Carlo Esposito

João Estêvão

Marco Faggella

Peter Fajfar

Harry Far

Michael Fardis

Charles Fernandez

Tiago Ferreira

Reza Filizadeh

Gabriele Fiorentino

Alessandro Flora

Paolo Foraboschi

Antonio Formisano

Maryam Foroutani

Giovanni Forte

Dora Foti

Sebastiano Foti

Stavroula Fotopoulou

Matthew Fox

Michalis Fragiadakis

Paolo Franchin

Andrea Franchini

Kevin Franke

Fabio Freddi

Kenji Fujii

Kohei Fujita

Marco Furinghetti

Andre Furtado

Ramon G. Drigo

Giammaria Gabbianelli

Carmine Galasso

Matija Gams

Charis Gantes

Antonio García-Jerez

Julian Garcia-Mayordomo

Paolo Gardoni

Sadegh Garivani

Dipendra Gautam

George Gazetas

Pierre Gehl

Fani Gelagoti

Katerina Genikomsou

Roberto Gentile

Ali Ghamari

Bahman Ghiassi

Amin Gholizad
Jayadipta Ghosh

Aga Giaralis

Giorgia Giardina

Rosario Gigliotti

Amir Gilani

Ivan Giongo

Katsuichiro Goda

Tatiana Goded

Jamie Goggins

Gideon Goitom

Rui Carrilho Gomes

Samo Gostič

Damian Grant

Russell Green

Mark Grigorian

Chuan Gu

Arianna Guardiola-Villora

Alessandra Gubana

Philippe Guéguen

Bertrand Guillier

Anxin Guo

Tong Guo

Chen Guoxing

Vittorio Gusella

Mohammad Ali

Hadianfard

Jerome Hajjar

Omer Halici

Qiang Han

Ufuk Hancilar

Pia Hannewald

Javad Hashemi

George Hatzigeorgiou

Richard Henry

Marijan Herak

Mohammed Hjiaj

Fabrice Hollender

Anne Hulsey

Iunio Iervolino

Afifa Imtiaz

Mehmet Inel

Bingunath Ingirige

Ioanna Ioannou

Tatjana Isakovic

Salvador Ivorra

Fatemeh Jalayer

Ana Beatriz Jaramillo

Emmanuel Javelaud

Jeena Jayamon
Liqiang Jiang

Zhiwei Jiang

Hsein Juang

James Kaklamanos

Afshin Kalantari

Georgios Kamaris

Vasileios Kamperidis

Alper Kanyilmaz

Onur Kaplan

Andreas Kappos

Faye Karababa

Faruk Karadogan

Ozlem Karagoz

Mary Karaveziroglou

Chris Karayannis

Mohammad Mehdi

Kashani

Evangelos Katsanos

Amir Kaynia

Athanasia Kazantzi

Shoma Kitayama

Mohsen Kohrangi

Maria Koliou

Martin Koller

Petros Komodromos

Susumu Kono

Stavroula Kontoe

Albert Kottke

Nicolas Kuehn

Sashi Kunnath

Ueda Kyohei

Pierre Labbe

Corinne Lacave

Vittoria Laghi

Carlo Lai

David Lallemant

Andreas Lampropoulos

Maria Lancieri

Luca Landi

Raffaele Landolfo

Dominik Lang

Nieves Lantada

Giovanni Lanzano

Oren Lavan

Milan Lazecky

Dawn Lehman

Jose Lemos

Nicos Lesgidis

Angeliki Lessi-Cheimariou 
Bo Li

Jianzhong Li

Zheng Li

Domenico Liberatore

Abbie Liel

Dimitrios Lignos

Henning Lilienkamp

Maria pina Limongelli

Asterios Liolios

Chengqing Liu

Wenguang Liu

Yong Liu

Marianna Loli

Domenico Lombardi

Mário Lopes

Francisco López-Almansa

Fernando Lopez-Caballero

Diego Lopez-Garcia

Susana Lopez-Querol

Paulo Lourenço

Jorge Macedo

Gregory Macrae

Srikanth Madabhushi

Giuseppe Maddaloni

Claudia Madiai

Gennaro Magliulo

Konstantia Makra

Christian Malaga-

Chuquitaype

Daniele Malomo

Ali Manafpour

Sujith Mangalathu

George Manos

Chrysanthos Maraveas

Basil Margaris

Alessandra Marini

Marko Marinković

Edoardo Marino

Olga Markogiannaki

Luis Martins

Y. Maruyam

Claudia Mascandola

Leonardo Massone

Fabio Mazza

Mirko Mazza

Ghyslaine McClure

Konstantinos

Megalooikonomou

Victor Melatti
Elena Mele

Bosco Melina

Giovanni Menichini

Panagiotis Mergos

Andrea Miano

Gabriele Milani

Zoran Milutinovic

Giovanni Minafò

Fabio Mistakidis

Euripidis Mistakidis

Stergios Mitoulis

Hiroe Miyake

Giuseppe Modoni

Fadzli Mohamed Nazri

Denis Moiriat

Vahid Mokarram

Elena Mola

Fabrizio Mollaioli

Gonzalo Montalva

Alvarado

Ricardo Monteiro

Luis Montejo Valencia

Jack Montgomery

Silvana Montoya-Noguera

Saber Moradi

Antonio Morales-Esteban

Paolo Morandi

Francesco Morelli

Konstantinos Morfidis

Mariantonietta Morga

Alireza Mortezaei

Ayman Mosallam

Mahdi Motagh

Carlos Moutinho

Harris Mouzakis

Dawid Mrozek

Aman Mwafy

George Mylonakis

Hosein Naderpour

Roberto Nascimbene

Maryam Nazari

Caterina Negulescu

Francesco Nex

Michele Nguyen

Thuong Anh Nguyen

Cecilia Nievas

Zeljana Nikolic

Ehsan Noroozinejad

Farsangi
Viviana Novelli

Camillo Nuti

Iolanda Nuzzo

Mitsu Okamura

Volkan Okur

Carlos Oliveira

Mario Ordaz

Gerard O'Reilly

Amirhossein Orumiyehei

Osman Ozbulut

Ali Özcebe

Yigit Ozcelik

Gokhan Ozdemir

Zuhal Ozdemir

Pelin Özener

Baki Ozturk

Marco Pagani

Alessandro Pagliaroli

Mehmet Palanci

Peng Pan

Yuxin Pan

Deepak Pant

S. Pantazopoulou

Chris Pantelides

Bartolomeo Pantò

Fabrizio Paolacci

Roberto Paolucci

Athanasios Papadopoulos

Georgios Papavasileiou

Ahsana Parammal Vatteri

Themelina Paraskeva

Fulvio Parisi

Yogita Parulekar

Edoardo Patelli

Florin Pavel

Alberto Pavese

Jelena Pejovic

Luca Pelà

Carlo Pellegrino

Gustavo Pereira

Rita Peres

Jesús Pérez-Herreros

Aymeric Perret du Cray

Daniele Perrone

Vicenzo Piluso

Rui Pinho

Dimitris Pitilakis

Kyriazis Pitilakis

Andre Plumier 


\begin{tabular}{|c|c|c|}
\hline Valerio Poggi & Hernán Santa Maria & Gülüm Tanırcan \\
\hline Daniel Pohoryles & V. Sarhosis & Tugrul Tankut \\
\hline Carmine Polito & Onur Seker & Nicola Tarque \\
\hline Yannis Politopoulos & Jean-Francois Semblat & Arturo Tena-Colunga \\
\hline Francesco Portioli & Karin Sesetyan & Gloria Terenzi \\
\hline Marco Preti & Salvatore Sessa & Solomon Tesfamariam \\
\hline Jean Herve Prevost & Barış Sevim & Paula Teves-Costa \\
\hline Jorge Proença & Anastasios Sextos & Nikos Theodoulidis \\
\hline Ioannis Psycharis & Ehsan Seyedi Hosseininia & Aris-Artemis Theophilou \\
\hline Rodolfo Puglia & Alper Sezer & KiranKumar Thingbaijam \\
\hline Luis G. Pujades & Benshun Shao & Eric Thompson \\
\hline Valentina Putrino & Bipin Shrestha & Patrick Tiong \\
\hline Virginio Quaglini & Manish Shrikhande & Dante Tolentino \\
\hline Patricio Quintana Gallo & Nigel Shrive & Roberto Tomasi \\
\hline Abdallah Rahmani & Zhan Shu & Alessandro Tombari \\
\hline Osman Ramadan & Vitor Silva & Cem Topkaya \\
\hline Antonio Ramos & Ana Simões & Selcuk Toprak \\
\hline Anirudh Rao & Konstantinos Skalomenos & Giacomo Torelli \\
\hline Ellen Rathje & Dario Slejko & Thanasis Triantafillou \\
\hline Julie Regnier & Chiara Smerzini & H. H. Tsang \\
\hline Philippe Renault & Eleni Smyrou & Grigorios Tsinidis \\
\hline Juan Reyes & Vladimir Sokolov & Georgios Tsionis \\
\hline Alfredo Reyes-Salazar & Farahnaz Soleimani & Panos Tsopelas \\
\hline Amir Rezaie & Pierre Sollogoub & Enrico Tubaldi \\
\hline Filipe Ribeiro & Xiaobin Song & Nerio Tullini \\
\hline Paolo Ricci & Stefano Sorace & Temel Türker \\
\hline Evi Riga & Siavash Soroushian & Jaroslav Vaculik \\
\hline Hugo Rodrigues & Luigi Sorrentino & Marco Valente \\
\hline Leonardo Rodrigues & Luis Sousa & Dimitrios Vamvatsikos \\
\hline Luís Rodrigues de Sousa & Romain Sousa & Humberto Varum \\
\hline Miranda & Berat Feyza Soysal & Graca Vasconcelos \\
\hline Mario Rodriguez & Enrico Spacone & Michalis Vassiliou \\
\hline Adrian Rodriguez-Marek & Robin Spence & Cuneyt Vatansever \\
\hline Fabio Romano & Daniele Spina & Ioannis Vayas \\
\hline Xavier Romão & Stefano Stacul & Ramon Verdugo \\
\hline Milad Roohi & Peter Stafford & Giulia Viggiani \\
\hline Leonardo Rossi & Sotiria Stefanidou & Susana Vilanova \\
\hline Maria Rota & Ioannis Stefanou & Manuela Villani \\
\hline Antonello Ruccolo & Jamison Steidl & Mabe Villar Vega \\
\hline Sergio Ruggieri & Dmitry Storchak & Marco Vona \\
\hline Jorge Ruiz-García & Aurel Stratan & Ali Reza Vosoughi \\
\hline Rajesh Rupakhety & Mark Stringer & Christos Vrettos \\
\hline Anna Saetta & Timothy Sullivan & Mladen Vucetic \\
\hline Erdal Safak & Armin Tabandeh & Kevin Walsh \\
\hline Dipti Ranjan Sahoo & Nima Tafazzoli & Bin Wang \\
\hline Jeff Salmon & Akihiro Takahashi & Gang Wang \\
\hline Thomas Salonikios & Shunsuke Takemura & Tao Wang \\
\hline Savvas Saloustros & Izuru Takewaki & Gang Wang \\
\hline M. Abdullah Sandikkaya & Masanori Tani & Xiaowei Wang \\
\hline
\end{tabular}




$\begin{array}{lll}\text { Graeme Weatherill } & \text { Susumu Yasuda } & \text { Farzin Zareian } \\ \text { Weiping Wen } & \text { Gunnur Yavuz } & \text { Irmela Zentner } \\ \text { Reeves Whitney } & \text { Mohammad Yekrangnia } & \text { Katerina Ziotopoulou } \\ \text { Liam Wotherspoon } & \text { Nozomu Yoshida } & \text { Maria Zucconi } \\ \text { Chenglong Wu } & \text { Yong Yuan } & \text { Abdullah Zulfikar } \\ \text { Yazhou Xie } & \text { Beatriz Zapico-Blanco } & \\ \text { Ahmet Yakut } & \text { Mehdi Zare } & \end{array}$

We apologize for any errors or inadvertent omissions.

Publisher's Note Springer Nature remains neutral with regard to jurisdictional claims in published maps and institutional affiliations. 